\title{
UMA NOVA DERIVAÇÃO DA TAXA DE DISSIPAÇÃO TURBULENTA PARA EVENTOS DE TURBULÊNCIA FRACA E BEM DESENVOLVIDA.
}

\author{
Lidiane Buligon ${ }^{* 2}$, Gervásio Annes Degrazia ${ }^{1}$, Charles Rogério Paveglio Szinvelski², Liliane \\ Moor ${ }^{1}$ \\ ${ }^{1}$ Departamento de Física, Universidade Federal de Santa Maria, RS-Brasil. \\ ${ }^{2}$ Departamento de Matemática, Universidade Federal de Santa Maria, RS-Brasil. \\ *buligon.1@ufsm.br
}

\section{RESUMO}

Neste trabalho é derivado uma nova forma funcional para a taxa de dissipação turbulenta válida para diferentes tipos de manifestações de turbulência presentes na CLP.

\section{ABSTRACT}

In this work is derived a new functional form for the turbulent dissipation rate valid for different types of turbulence in the PBL.

\section{INTRODUÇÃO.}

Uma relação importante no estudo de um escoamento turbulento é expressa pela forma funcional da taxa de dissipação de energia turbulenta. Esta relação, além de descrever os parâmetros fundamentais que determinam os diferentes aspectos da turbulência, permite simular o campo de concentração de contaminantes pelo emprego de modelos de dispersão estocásticos lagrangianos. Normalmente, uma equação baseada na função de autocorrelação lagrangiana exponencial (Taylor, 1921) é válida para uma turbulência bem desenvolvida, consequentemente não pode ser aplicada em eventos de meandro caracterizados pela presença de uma turbulência fraca (Afonssi et al., 2005; Degrazia et al., 2008).

Tennekes (1982) derivou a seguinte relação fundamental para a razão de dissipação turbulenta $\varepsilon$,

$$
\varepsilon=\frac{2}{C_{0}} \frac{\sigma_{v}^{2}}{\mathrm{~T}_{\mathrm{L}_{\mathrm{v}}}},
$$

Relações funcionais, tais como a Eq. (1), podem ser usadas na determinação da constante de Kolmogorov e nas parametrizações turbulentas aplicadas em modelos de dispersão estocásticos lagrangianos (Yeung, 2002).

O objetivo deste estudo é derivar uma forma funcional para a taxa de dissipação turbulenta que seja válida para diferentes tipos de manifestações de turbulência presentes na CLP. Do ponto de vista físico, será derivada uma relação fundamental geral descrevendo a dissipação turbulenta associada ao fenômeno de meandro do vento horizontal.

\section{DERIVAÇÃO DA RELAÇÃO FUNDAMENTAL (1) PARA EVENTOS DE TURBULÊNCIA FRACA E BEM DESENVOLVIDA.}

Degrazia propôs a seguinte relação matemática para representar a função de autocorrelação lagrangiana para a turbulência na CLP (Pasquill \& Smith, 1983 ; Phillips \& Panofsky, 1982). 


$$
\rho_{L_{v}}(t)=\frac{\cos (q t)}{(1+p t)^{2}}
$$

onde $\quad \mathrm{p}=\frac{1}{\left(1+m^{2}\right) T_{L}}, \quad \mathrm{q}=\frac{m}{\left(1+m^{2}\right) T_{L}}$ e $m$ é uma quantidade adimensional que controla a frequência de oscilação do meandro do vento horizontal .

Utilizando a Equação de Taylor para a variância espacial latera,

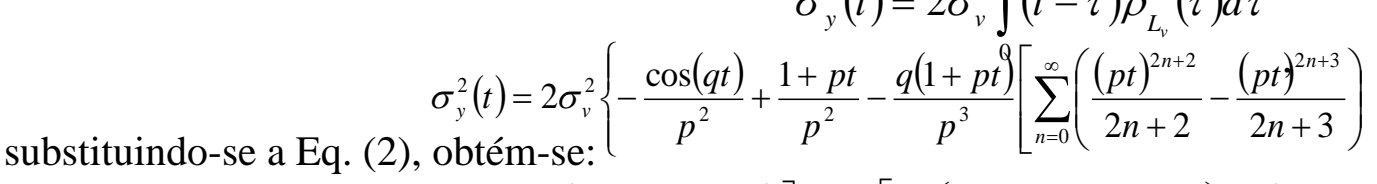

$$
\left.\left.\sum_{k=0}^{\infty}\left((-1)^{n} \frac{m^{2 k+1}}{(2 k+1) !}\right)\right]-\frac{1}{p^{2}}\left[\sum_{n=0}^{\infty}\left(\frac{(p t)^{2 n+1}}{2 n+1}-\frac{(p t)^{2 n+2}}{2 n+2}\right) \sum_{k=0}^{\infty}\left((-1)^{n} \frac{m^{2 k}}{(2 k) !}\right)\right]\right\}
$$

Neste ponto, fazendo-se uma expansão em Série de Maclarin para $t<T_{L}$, e truncando

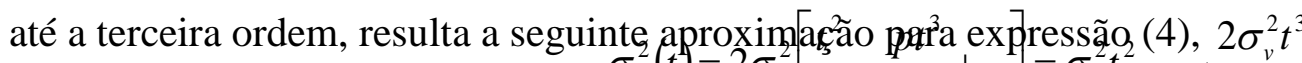

$$
\sigma_{y}^{2}\left(t^{\prime}\right)=2 \sigma_{v}^{2}\left[\frac{5}{2}-\frac{p 4}{3}+\ldots\right]=\sigma_{v}^{2} t^{2}-\frac{2 \sigma_{v}^{2} t}{3\left(1+m^{2}\right) T_{L}}+\ldots
$$

Considerando-se o Modelo de Difusão Estatístico de Taylor na representação espectral, fica evidente que o termo de correção negativo está associado aos graus de liberdade turbulentos do subintervalo inercial do espectro de energia. Desta maneira, seguindo a derivação proposta por Ternekeş (1982) pode-se escrever,

$$
\sigma_{y}^{2}(t)=\sigma_{v}^{2} t^{2}-\frac{c^{2}}{6} t^{3}
$$

Da comparação direta da Eq. (5) com a Eq. (6), resulta uma fórmula funcional geral para a razão de dissipação turbulenta, escrita na seguintøforma: $4 \frac{\sigma_{v}^{2}}{C_{0}}=\frac{\sigma_{v}^{2}}{\left(1+m^{2}\right)} \frac{C_{0} T_{L}}{C_{0}}$

(i) $\mathrm{m}=\underset{\text { Assim se: }}{0 \underset{C_{0} T_{L}}{\Rightarrow}} \boldsymbol{\varepsilon}=4 \frac{\sigma_{v}^{2}}{C_{0}}$

(ii) $\mathrm{m}=1 \Rightarrow \varepsilon=2 \frac{\sigma_{v}^{2}}{C_{0} T_{L}}$

(iii) $\mathrm{m} \rightarrow \infty \Rightarrow \varepsilon \rightarrow 0$.

Do ponto de vista físico, a nova relação fundamental dada pela Eq. (7) mantém a premissa básica contida na Eq. (1), ou seja, a razão de dissipação da turbulência é proporcional à energia disponível e inversamente proporcional a escala de tempo associada aos turbilhões que contém a energia principal do campo turbulento. Apesar disso, surge nesta nova relação o parâmetro $p$ que relaciona $\varepsilon$ ao fenômeno de meandro (turbulência fraca). Da relação entre $p$ e $m$, pode-se ver que à medida que $m$ aumenta e o fenômeno de meandro começa a se manifestar, a taxa de dissipação turbulenta diminui. Este comportamento é 
fisicamente razoável, uma vez que as oscilações de baixa freqüência são relacionadas a números de onda muito menores do que aqueles associados à dissipação molecular.

\section{CONCLUSÃO.}

Neste estudo foi representada uma expressão alternativa para a taxa de dissipação turbulenta para a CLP. Esta nova expressão leva em conta os efeitos turbulentos e o comportamento de onda associado às oscilações de baixa freqüência observadas no fenômeno de meandro. A diferença desta relação para o resultado clássico, normalmente aceito para a taxa de dissipação, é a presença de um parâmetro que controla a freqüência de oscilação do meandro do vento horizontal.

\section{REFERÊNCIAS.}

ANFOSSI, D. et al. An analysis of sonic anemometer observations in low wind speed conditions. Boundary-Layer Meteorology, v. 114, n. 1, p. 179-203, 2005.

DEGRAZIA, G. A. et al. Turbulence dissipation rate derivation for meandering occurrences in a stable planetary boundary layer. Atmospheric Chemistry and Physics, v. 8, n. 6, p. 1713-1721, 2008.

PASQUILL, F and SMITH, F. B. Atmospheric diffusion. E. Horwood, 437, 1983.

PHILLIPS, P. and PANOFSKY, H.A. A re-examination of lateral dispersion from continuous sources, Atmospheric Environment. 16, 1851-1860, 1982.

TAYLOR, G. I. Diffusion by continuous movements, Proc. London Math. Soc. 20, 196$211,1921$.

TENNEKES, H. Similarity relations, scaling laws and spectral dynamics. In: Atmospheric turbulence and air pollution modelling. Springer Netherlands, 37-68, 1982.

YEUNG, P. K. Lagrangian investigations of turbulence. Annual review of fluid mechanics, v. 34, n. 1, p. 115-142, 2002. 